\title{
Activation of the ROS/CncC and 20-Hydroxyecdysone Signaling Pathways is Associated with Xanthotoxin-Induced Tolerance to $\lambda$-Cyhalothrin in Spodoptera litura
}

Kai Lu', Yimin Li ${ }^{\text {a }}$, Yibei Cheng ${ }^{a}$, Wenru Lia ${ }^{a}$ Bixue Zenga, Chengzhen Gua Rensen Zeng ${ }^{b}$, Yuanyuan Song ${ }^{\text {* }}$

${ }^{a}$ State Key Laboratory of Ecological Pest Control for Fujian and Taiwan Crops, College of Life Sciences, Fujian Agriculture and Forestry University, Fuzhou, 350002, China

${ }^{\mathrm{b}}$ Key Laboratory of Ministry of Education for Genetics, Breeding and Multiple Utilization of Crops, College of Agriculture, Fujian Agriculture and Forestry University, Fuzhou, 350002, China

\section{* Corresponding Author}

Prof. Yuanyuan Song

E-mail address: yyuansong@,fafu.edu.cn

Tel.: +86-0591-83789272.

Running head: Xanthotoxin enhances insecticide tolerance 


\section{Supporting Information}

Table S1. Primers used in this study.

\begin{tabular}{|c|c|}
\hline Primer name & Primer sequence $\left(5^{\prime}-3^{\prime}\right)$ \\
\hline \multicolumn{2}{|l|}{ For qRT-PCR } \\
\hline$A h R-\mathrm{qF}$ & GAAGTGGCCCCCACGTATCA \\
\hline$A h R$-qR & CGGTCCAGGCACTACGGAAG \\
\hline$H N F 4-\mathrm{qF}$ & GACGGCTGCAAGGGTTTCTTC \\
\hline$H N F 4-\mathrm{qR}$ & TCCCGCTTTGAAGCATTTCCT \\
\hline HR96-qF & ATCCCCCACTGCCAAGAACA \\
\hline HR96-qR & CCTCGAACGAGCGGATGAAG \\
\hline$F T Z-F 1-\mathrm{qF}$ & TTACGCAGCGGTTCCAGTCA \\
\hline$F T Z-F 1-\mathrm{qR}$ & CAAAGCTGGGCCTGGTGAAG \\
\hline CYP302A1-qF & TGGCAGCCTTGTTCGTGAAA \\
\hline CYP302A1-qR & CTCCTTGCTGGGAACCGATG \\
\hline CYP306A1-qF & CTGAGGATGGCGAGGTGGAC \\
\hline CYP306A1-qR & GGGATTCCGACGGGCACTAT \\
\hline CYP307A1-qF & GGATTTCCTGCCTTGGCTCA \\
\hline CYP307A1-qR & CAGGCCCTTCGATGTCCAAA \\
\hline CYP314A1-qF & CCTGGGAAGCGATTTGTGGA \\
\hline CYP314A1-qR & TAACCGCAGCGACACTGGAG \\
\hline$C Y P 315 A 1-\mathrm{qF}$ & CGTTGCACCGTTTTTGACCA \\
\hline CYP315A1-qR & ATTGCGGTCCCAACGGTAAG \\
\hline$E c R-\mathrm{qF}$ & AAGCCTTTGCCGGAGGATGT \\
\hline$E c R$-qR & GCGTCTGGAAACCTCCGTTG \\
\hline$U S P-\mathrm{qF}$ & CGATGGTGGCTGATCCCACT \\
\hline$U S P-q R$ & TTGAGACAGGGGCACGGAAC \\
\hline \multicolumn{2}{|c|}{ For dsRNA synthesis } \\
\hline$E c R-F i$ & ggatcctaatacgactcactatagggCAACAGCCCCAGCAGACTCA \\
\hline$E c R-R i$ & ggatcctaatacgactcactatagggCGTGGCAACACCTCGTTCTG \\
\hline$U S P-F i$ & ggatcctaatacgactcactatagggGACGTACGCGTGTCGTGAGG \\
\hline$U S P-R i$ & ggatcctaatacgactcactatagggAGTTGTGGTGGCGATGTGGA \\
\hline
\end{tabular}

F: forward primer; R: reverse primer. Lowercase letters indicate the $\mathrm{T} 7$ promoter sequence. 
CYP6AE43, E=89.82\%

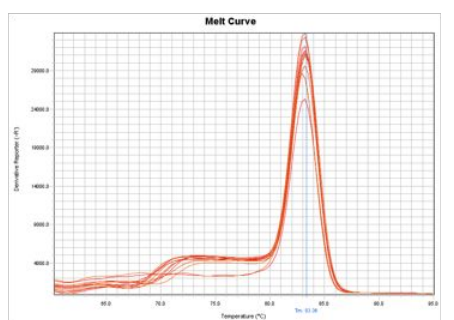

CYP6AE50, E=89.88\%

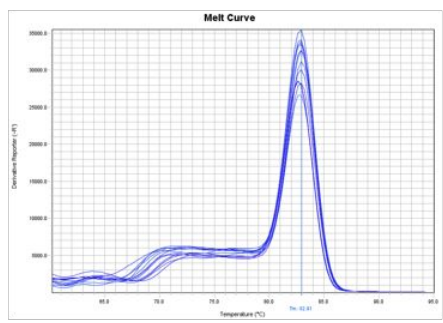

CYP6AE97, E=106.16\%

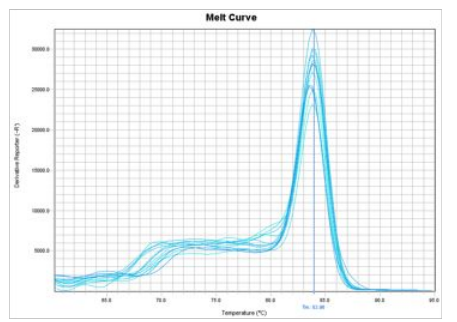

CYP6B 48, E $=98.01 \%$

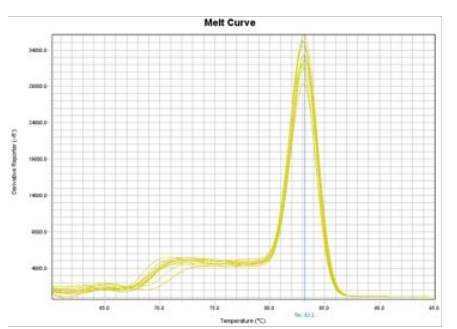

CYP321A8, E=87.36\%

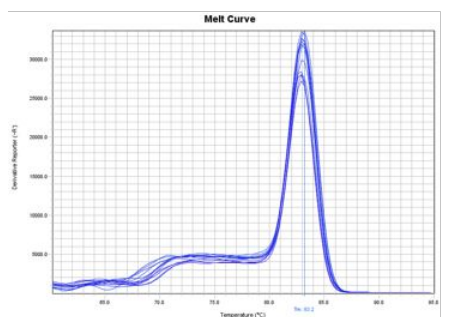

$G S T-S 1, \mathrm{E}=90.62 \%$

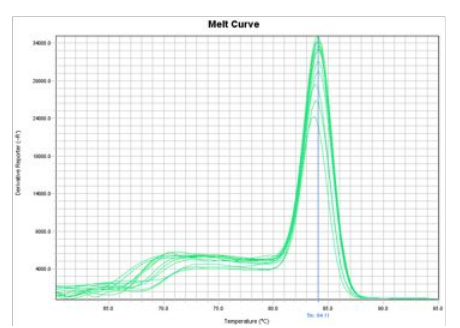

CYP6AE47, E=92.84\%

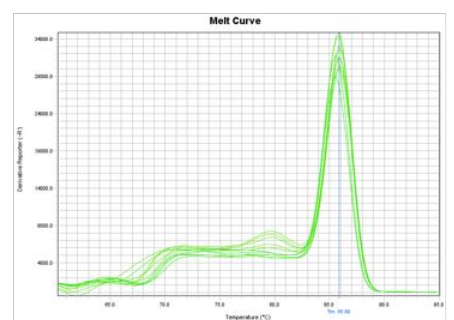

CYP6AE68, E=89.47\%

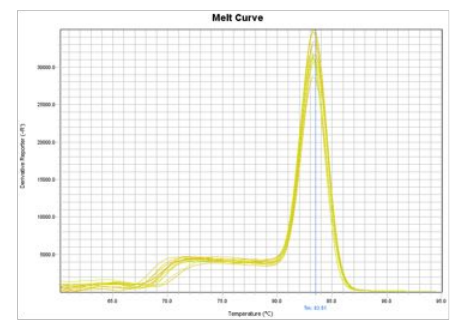

CYP6B46, E $=109.94 \%$

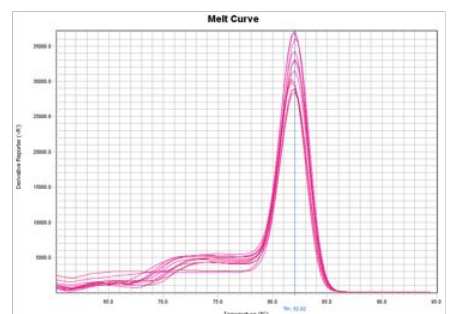

CYP6B58, E=99.71\%

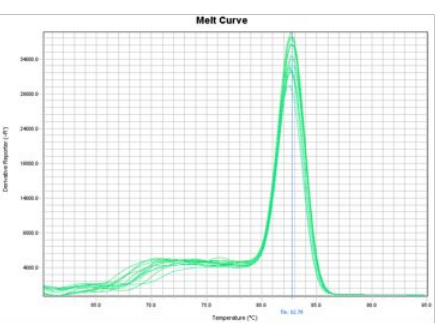

CYP321A10, $\mathrm{E}=94.91 \%$

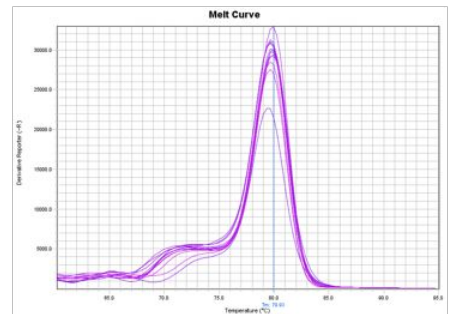

$G S T-S 2, \mathrm{E}=86.9 \%$

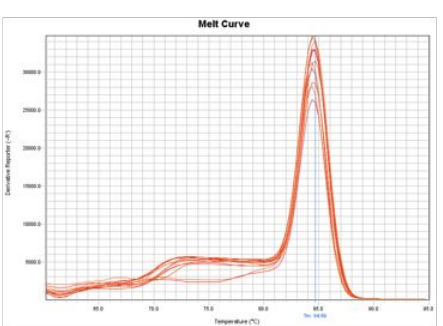

CYP6AE48, E=100.9\%

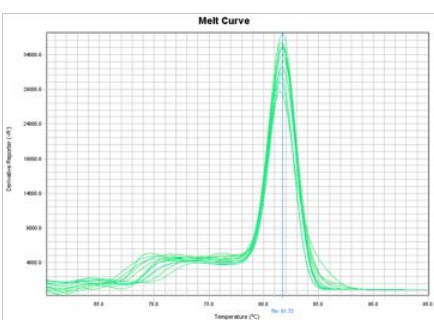

CYP6AE70, E=97.53\%

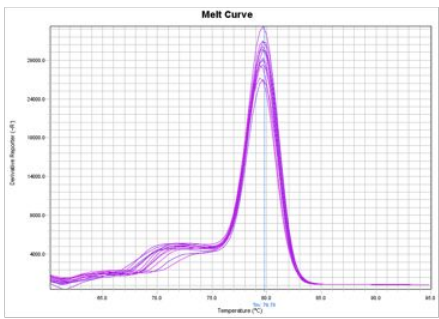

CYP6B47, E=90.14\%

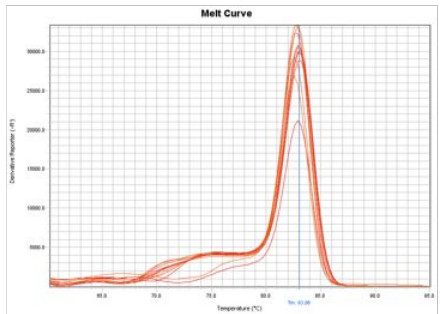

CYP321A7, E $=86.48 \%$

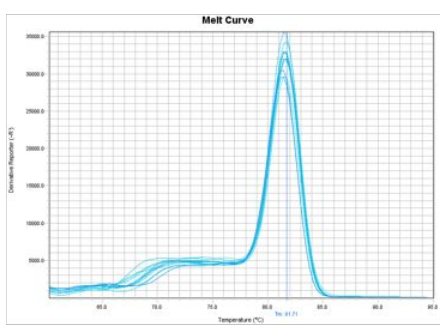

CYP321A12, E=94.47\%

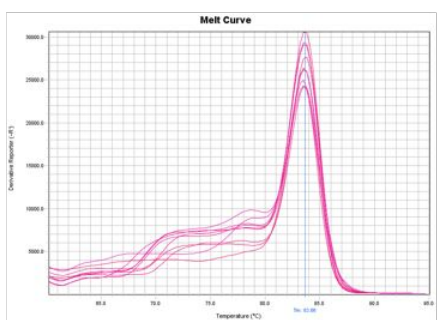

$G S T-S 3, \mathrm{E}=108.52 \%$

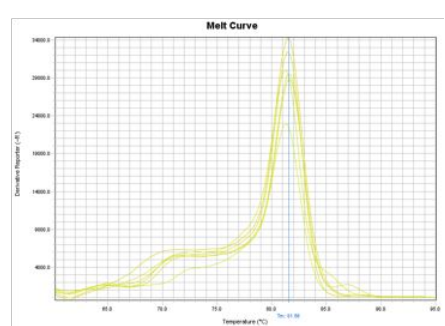



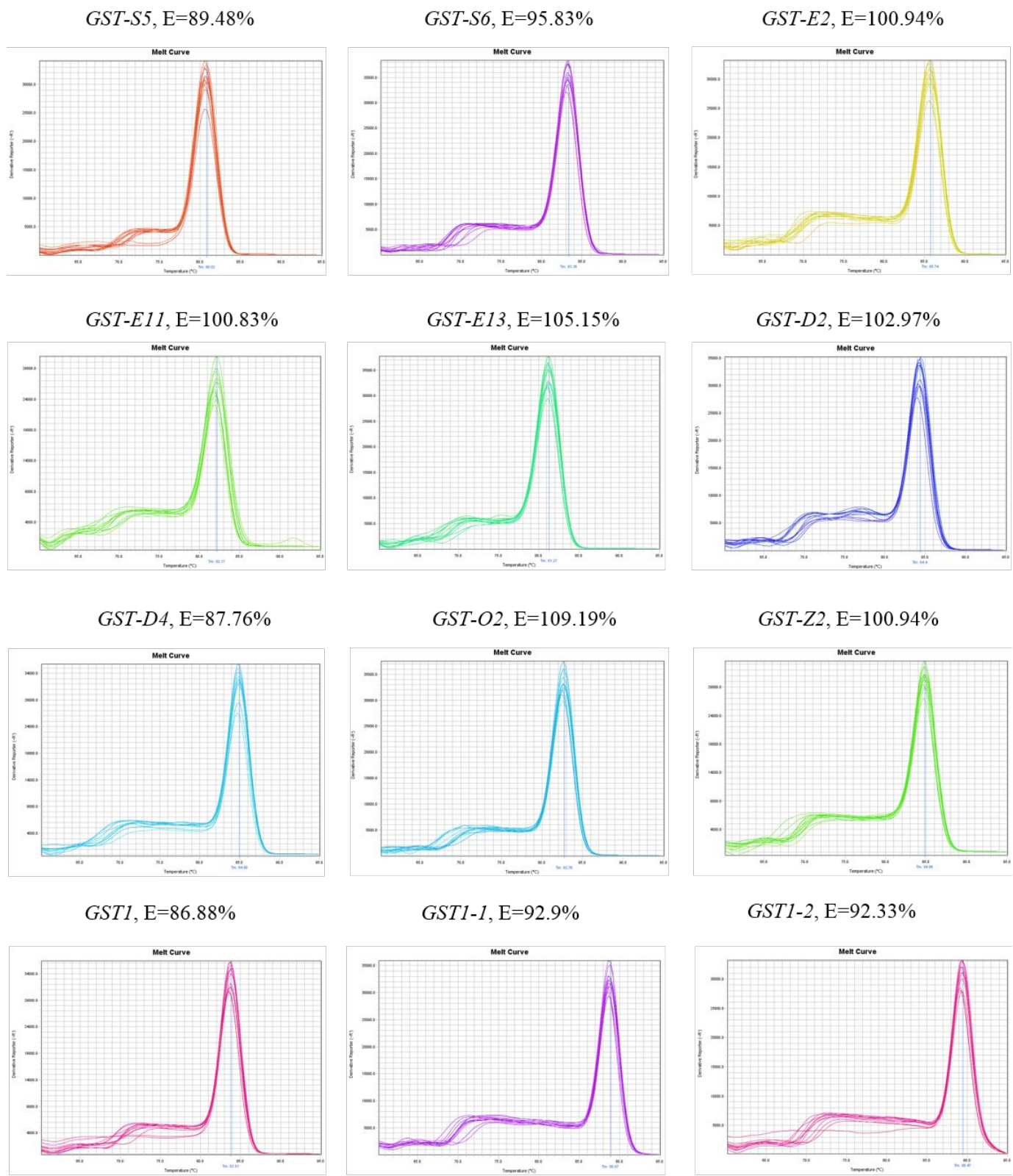

GST1-2, E=92.33\%

$U G T 42 C 2, \mathrm{E}=102.24 \%$

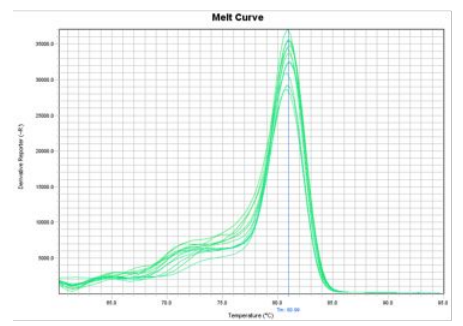

UGT40M3, $\mathrm{E}=107.54 \%$
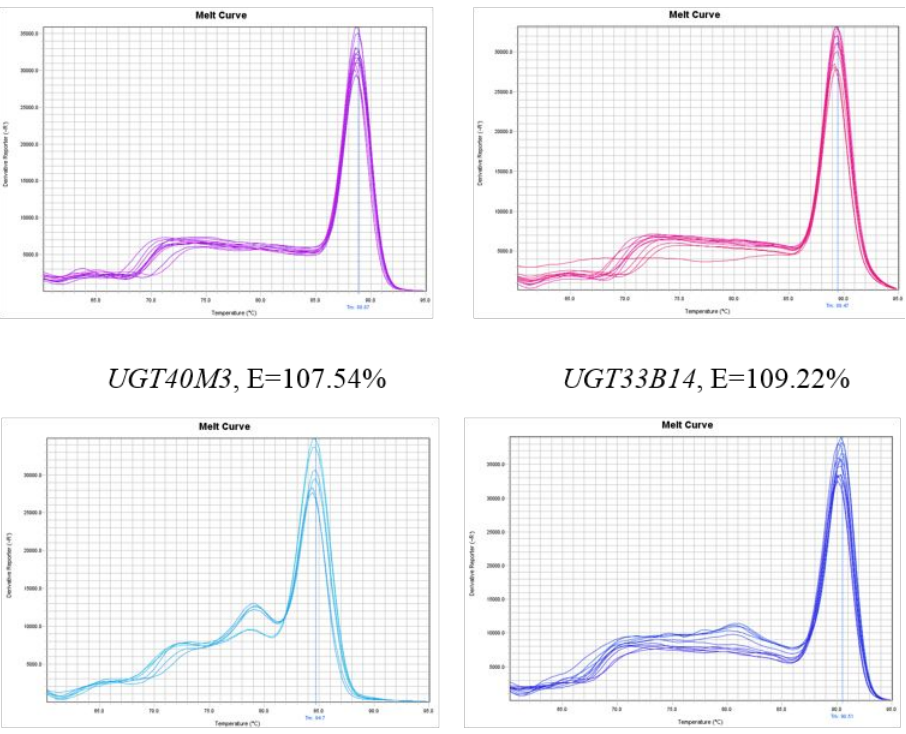

UGT33B14, $\mathrm{E}=109.22 \%$

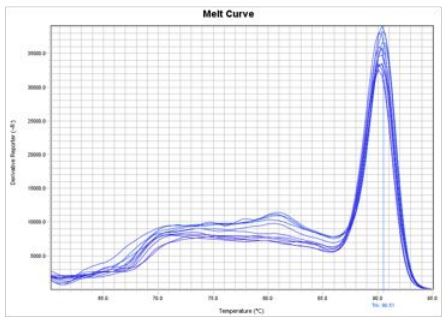

UGT4OF $4, \mathrm{E}=98.29 \%$
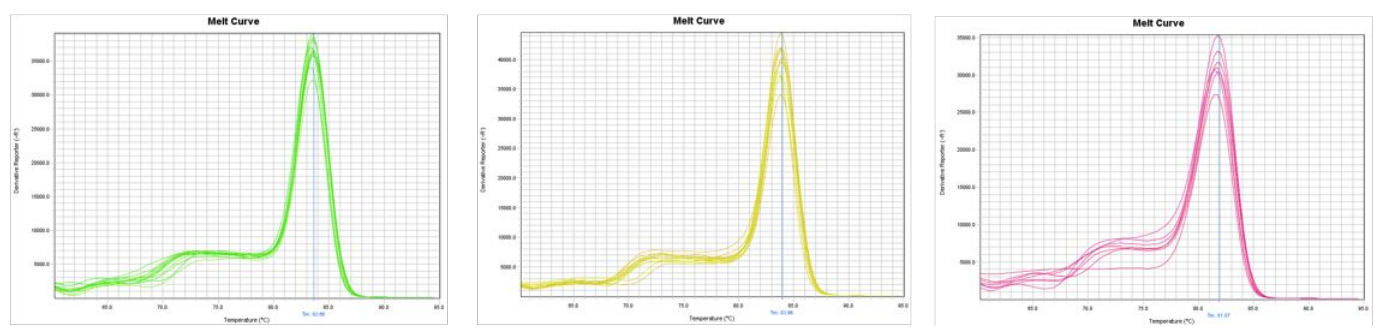

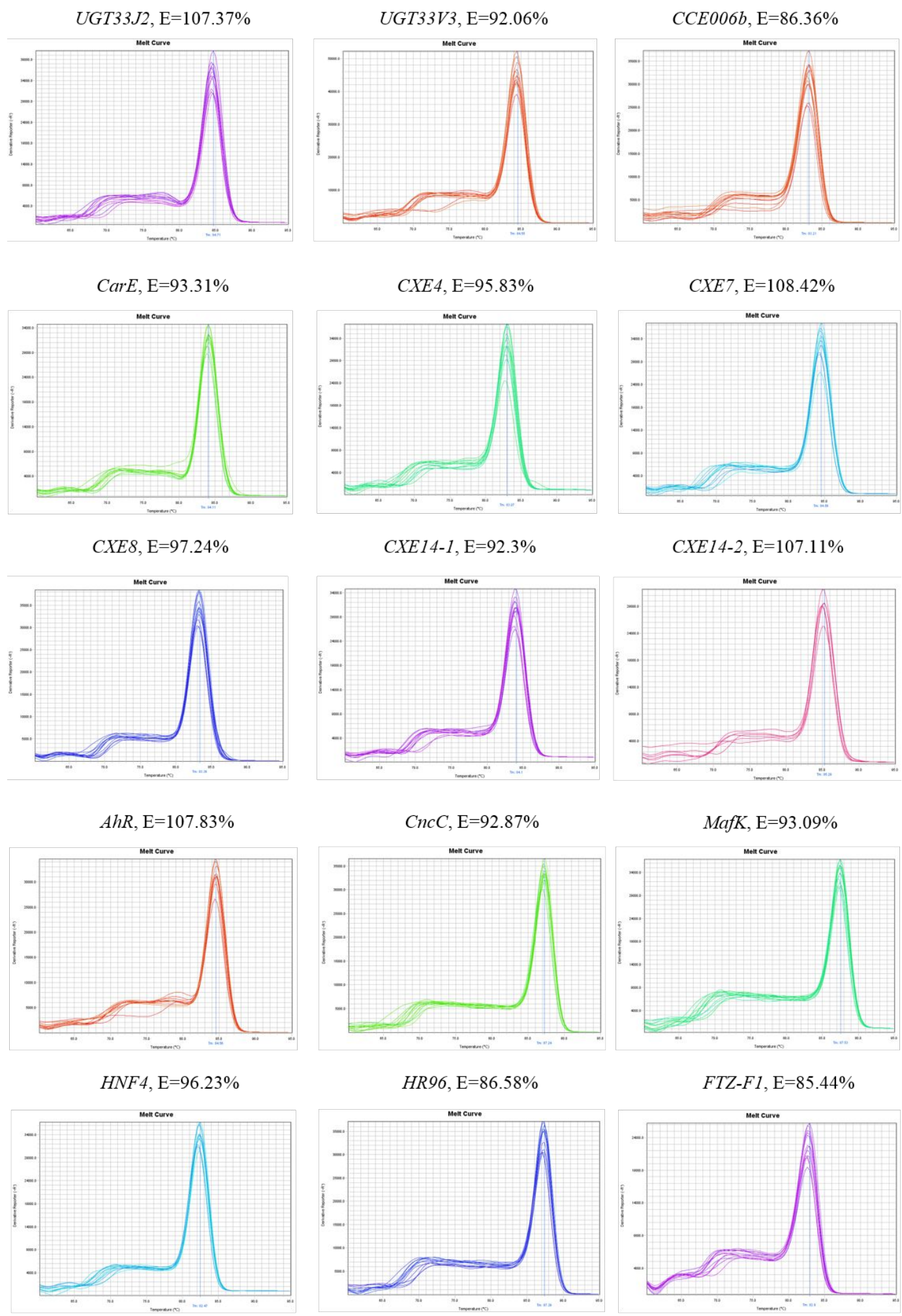

$F T Z-F 1, \mathrm{E}=85.44 \%$
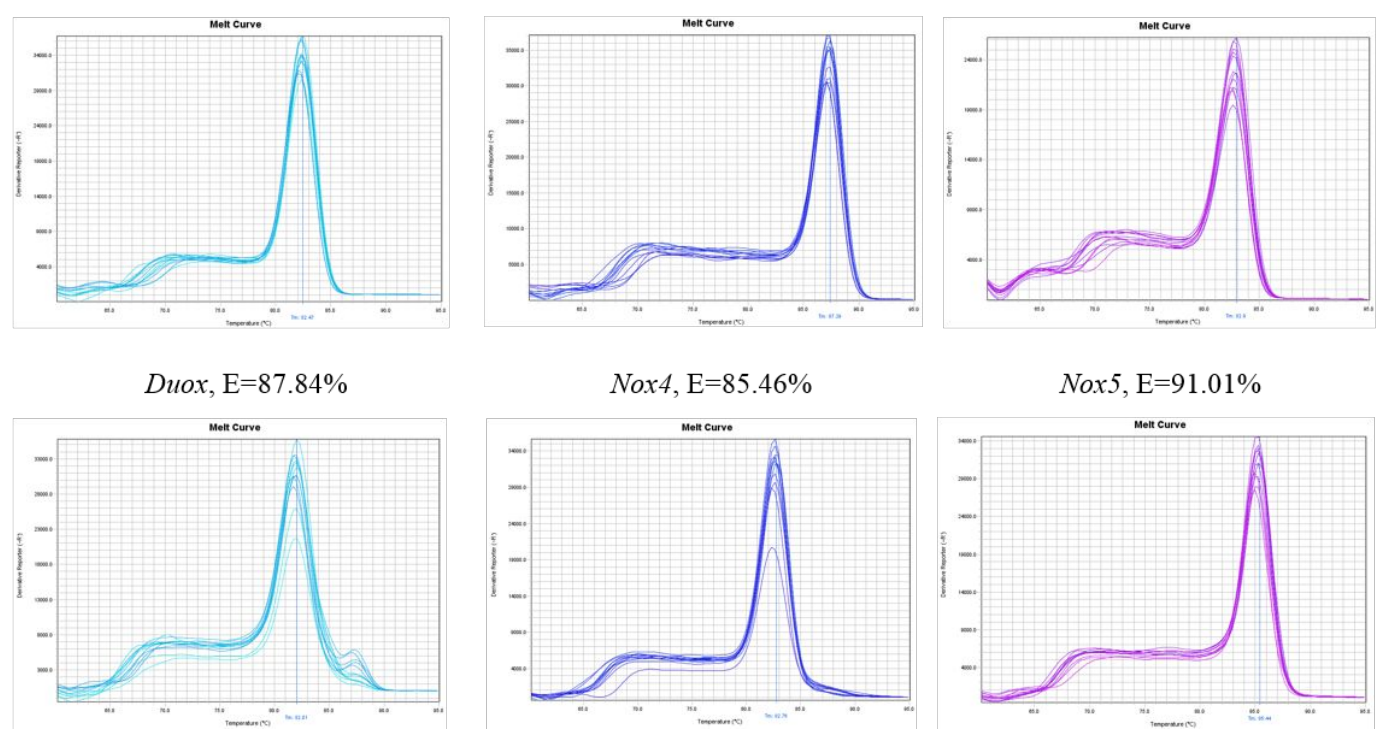
$E c R, \mathrm{E}=93.58 \%$

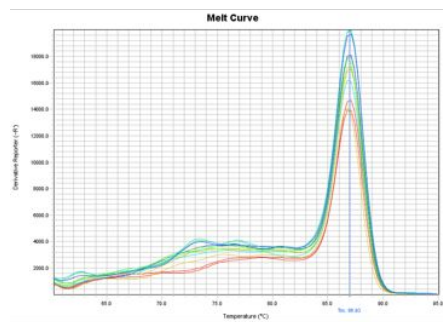

CYP306A1, $\mathrm{E}=95.69 \%$

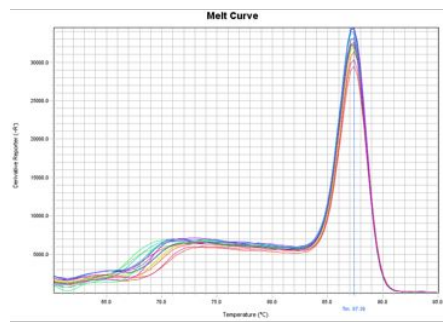

CYP315A1, $\mathrm{E}=89.35 \%$

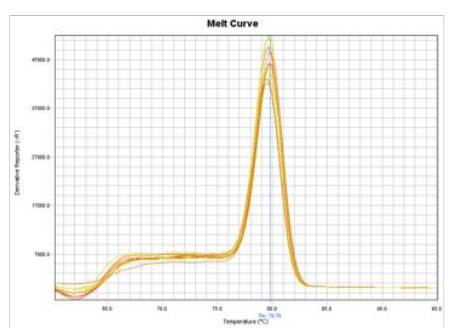

$U S P, \mathrm{E}=89.87 \%$

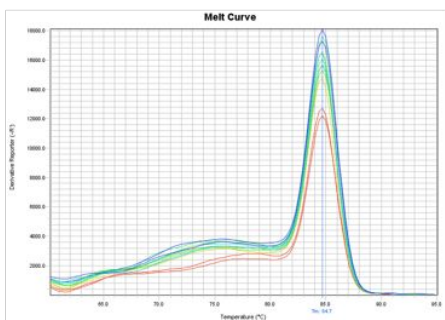

CYP307A1, $\mathrm{E}=103.25 \%$

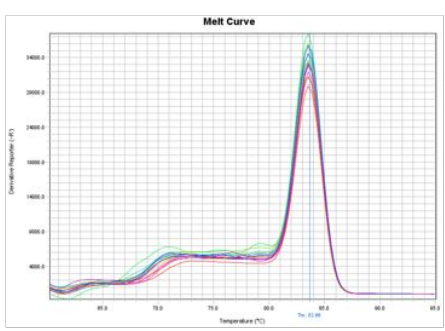

$E F-1 a, \mathrm{E}=96.58 \%$

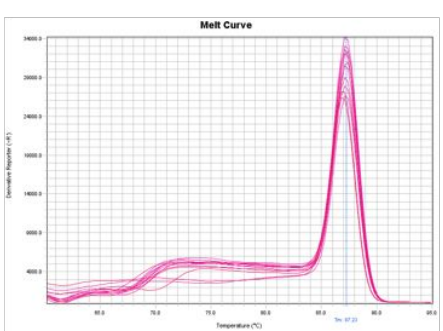

CYP302A1, $\mathrm{E}=109.15 \%$

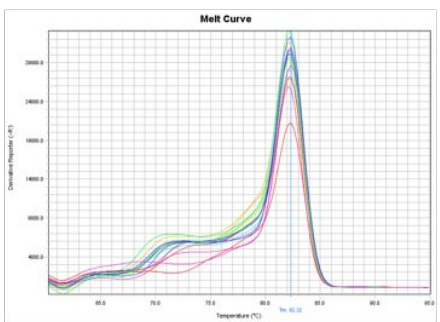

CYP314A1, E=102.92\%

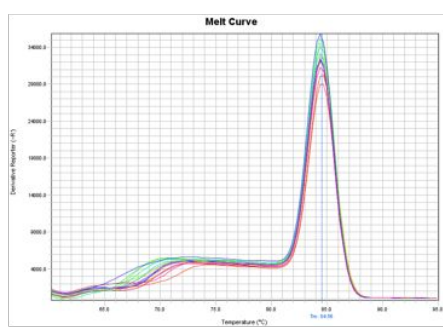

Figure S1. The melting curves and PCR amplification efficiencies of all primer sets. 